\title{
The role of family therapy in the management of schizophrenia: challenges and solutions
}

REVIEW

\author{
This article was published in the following Dove Press journal: \\ Neuropsychiatric Disease and Treatment \\ 14 January 2015 \\ Number of times this article has been viewed
}

\author{
Alejandra Caqueo-Urízar ${ }^{1, *}$ \\ Mar Rus-Calafell2,* \\ Alfonso Urzúa ${ }^{3}$ \\ Jorge Escudero' \\ José Gutiérrez-Maldonado 4 \\ 'Departamento de Filosofía y \\ Psicología, Universidad de Tarapacá, \\ Arica, Chile; ${ }^{2}$ Department of Social \\ Psychiatry, Institute of Psychiatry, \\ King's College London, London, UK; \\ ${ }^{3}$ Alfonso Urzúa Morales, Escuela \\ de Psicología, Universidad Católica \\ del Norte, Antofagasta, Chile; \\ ${ }^{4}$ Departamento de Personalidad, \\ Evaluación y Tratamientos \\ Psicológicos, Universidad de \\ Barcelona, Barcelona, Spain \\ *These authors have contributed \\ equally to this work
}

\begin{abstract}
Family interventions for schizophrenia have been amply demonstrated to be effective and are recommended by most of the international clinical guidelines. However, their implementation in the clinical setting as well as in treatment protocols of patients with psychosis has not been fully achieved yet. With the increasing deinstitutionalization of patients, family has begun to assume the role of care performed by psychiatric hospitals, with a high emotional cost for caregivers as well as the recognition of burden experiences. Families have been the substitute in the face of the scarcity of therapeutic, occupational, and residential resources. For this reason, the viability of patients' care by their families has become a challenge. This article aims to discuss the most important aspects of family interventions, their impact on families, and the most important challenges that need to be overcome in order to achieve well-being and recovery in both patients and caregivers.
\end{abstract}

Keywords: family intervention, schizophrenia, caregivers, quality of life, therapy

\section{Introduction}

Schizophrenia is a serious mental disorder that has a significant impact not only on patients suffering from the disorder but also on their families. ${ }^{1}$ The end of traditional institutional care within psychiatric hospitals has led to an emphasis on the importance of emotions and affections within the family and the recognition of the high level of burden on relatives. The social and emotional implications for families and patients with schizophrenia are harmful, resulting in impairment of social and occupational functioning. ${ }^{2}$ From the onset of the disorder, caregivers spend a lot of time worrying about their relatives' well-being and future care, ${ }^{3}$ neglecting their own care. The uncertainty about diagnosis, prognosis, and adequate treatment can trigger high levels of anxiety and stress within the family and all this contributes to caregivers burden. ${ }^{4}$ In addition, families must adjust very quickly to this new role of "informal care", ${ }^{5}$ for which they often feel unprepared. ${ }^{6}$

It is estimated that in Western countries, between $50 \%$ and $80 \%$ of patients with schizophrenia are in close contact with their relatives and friends. ${ }^{7}$ When the patients return to the community after an admission or first contact with the mental health services, their relatives are the main support system, even more than the medical staff. ${ }^{8}$ Muela and Godoy indicated that there is "something in the family interaction that seems to cause a relapse of the patient and seems to influence the course of the disease, instead of being the origin of the same". ${ }^{9}$ Following this, it seems reasonable to dedicate research and clinical resources to determine to a greater extent the needs of caregivers in order to improve the family environment and, consequently, the patient's recovery.
Correspondence: Mar Rus-Calafell

Department of Social Psychiatry, Institute of Psychiatry, King's College London, De Crespigny Park, SE5 8AF London, UK

Tel +442078485087

Fax +44 207277 I 462

Email maria.rus-calafell@kcl.ac.uk 
Taking on a caring role is at an important cost for the person, who loses a series of opportunities for his personal and working growth. Thus, the establishment of a psychotic diagnosis often has a negative impact on the social networks of caregivers, rendering them isolated and lonely, with feelings of stigmatization, particularly if their relative has been involved in antisocial behavior. ${ }^{10}$ Caring for these patients in the family has a number of consequences: individual social and economic changes known as caring effects. The physical health and mental health of key caregivers have been seen as the most significant factors that determine the level of burden of caregivers. ${ }^{8}$

Deinstitutionalization, urbanization, and the establishment of evidence-based interventions, driven by economic and political factors, are important factors that affect the present and the future of family interventions for schizophrenia. For the purposes of the present article, we will critically discuss how these interventions impact on the quality of life of both patients and their families and what the most important challenges are for the clinical and research community in order to improve the positive impact of these interventions on caregivers.

\section{Impact on families}

The main caregiver is the individual, generally a member of the patient's family, who spends most of his/her time caring for the patient, supplying support, and checking medication and other aspects of the patient's daily life. caregivers frequently report feelings of guilt and blame (eg, feeling that they failed to help their relatives soon enough or that they missed key signs and symptoms indicative of the illness). ${ }^{11}$ This situation often implies the experience of being overloaded and its incidence rate can be generalized for relatives from different countries; however, the main differences with regards to the experience of burden correspond to better and wider access to sanitary and economic resources available for these caregivers. In third-world countries or in developing countries, economic burden plays an outstanding role in the relatives' quality of life. The lack or shortage of psychiatrists, day hospitals, and access to pharmacological treatment produce a significant preoccupation in these relatives. The patient's sex and age do not appear to have an effect on families' overheads, although educational level may have an influence. ${ }^{12,13}$ Three studies showed that about one-fifth to one-third of the members of the family reported difficulties and distant relations with the rest of the family group because of the existence of mental illness. ${ }^{14-16}$ Some studies on burden have shown that caregivers of psychiatric patients are highly burdened by the task; this burden is both objective and subjective. ${ }^{17-22}$ Objective burden refers to "the symptoms of the caregivers and sociodemographic characteristics, but also to changes in the daily routine, family and social relations, work, leisure and physical health", ${ }^{23}$ On the other hand, the subjective burden would be "the result of subjective discomfort and problems of health". ${ }^{24}$ Studies on family intervention have managed to reduce the burden experienced by these caregivers; nevertheless, psychoeducational interventions are not fully integrated into the treatment in mental health services. ${ }^{25-28}$ This relationship between caregivers and the mental health services would allow the establishment of social support networks, which have been related to a reduction in caregivers' physical and mental burden.

Therefore, experiencing burden will reduce caregivers' quality of life. Important studies have shown psychopathological risk and damage in the quality of life of many caregivers, especially women..$^{21,29-32}$ The more burden these relatives experience, the worse their quality of life will be. ${ }^{33,34}$ Although there is no consensus in the definition of this concept, the World Health Organization defines it as an individual's perception of their position in life in the context of the culture and value systems in which they live and in relation to their goals, expectations, standards, and concerns. ${ }^{35}$ Thus, the quality of life would be presented as the perceived level of well-being derived from the assessment made by caregivers with regard to various elements in different dimensions of their lives. ${ }^{36}$ Decrease in vitality and health (developing depression and anxiety), decrease in interpersonal and social interactions, and even body pain are the results of a low quality of life. ${ }^{37-41}$ However, some variables can increase the caregivers' perception of quality of life, for example, having social support or getting a job outside the home. Daily activities outside the home imply a certain degree of freedom to the caregivers because they feel they are useful or that they have other functions within the community. This cognitive and emotional "freedom" can increase their level of satisfaction and ameliorate the negative impact of the disorder. ${ }^{17,22,42-44}$

However, not all the literature in relation to the care of psychiatric patients shows negative results in the caregivers. Currently, new lines of research have emerged showing the positive aspects of the experience of caring. ${ }^{45,46}$ Although there is still no clear definition regarding positive caregivers experience, this is related to three main topics. ${ }^{47-49}$ (a) Caregivers' satisfaction: The definition has changed over the years, but it can be thought of as the result of caregiving experiences that give life "positive flavor" ${ }^{45,50}$ (b) Caregivers" gain: This is 
defined as the extent to which the caregiving role is appraised to enhance an individual's life and be enriching space. ${ }^{51}$ (c) Caregivers' experience: This includes the establishment of a good relationship with the patient and positive personal outcomes. ${ }^{45,47}$ Despite the relative clarity that seems to exist regarding the impact of schizophrenia on family members, it is necessary to consider the customs and traditions of each family group, as it has been found that cultural patterns can identify not only the type of behaviors established with their ill family member but also the burden related to the relationship between them. Therefore, it is suggested that efforts should be made to collect sensitive aspects of the culture of the families, as they can influence for better or worse the ability to cope with the disorder. Therefore, future cross-cultural comparisons are needed. ${ }^{52}$

\section{Family interventions}

Although there is not a unique approach for family interventions, evidence-based family therapies usually include psychoeducation, stress reduction, emotional processing, cognitive reappraisal, and structured problem solving. Intervention consists of a combination of psychotherapeutic strategies for working with the relatives of people who suffer from psychosis, and it aims to develop a collaborative relationship between the family and the treatment team in order to help patients make progress toward recovery ${ }^{53}$ Interventions are always delivered by mental health professionals but can differ in theoretical orientation, modality, or length.

More than 50 controlled trials have been conducted to test the efficacy of these kinds of interventions. ${ }^{53,54}$ Principal findings have proven that family therapy has a positive impact on the patient's recovery, with a significant reduction in readmission and relapses, as well as an improvement in their social functioning. ${ }^{55}$ Furthermore, Pilling et $a{ }^{56}$ found a link between these interventions and adherence to medication. Only a few studies have reported that family interventions improve experience of care and the commitment to the caregiving role. ${ }^{25,57}$ Although all these studies on family intervention have shown a series of outcome measures, both intervention programs and studies' goals usually focus mainly on patients rather than caregivers.

Several studies have shown encouraging findings after the application of psychoeducational programs for patients with schizophrenia and their families. ${ }^{6,29-31}$ A study by Magliano et al noted a clear improvement in the social functioning of caregivers and also their levels of burden, with similar findings in different European countries. ${ }^{32}$ In addition, some of these intervention programs have been proven effective in increasing caregivers' perception of self-efficacy and social support, as well as levels of satisfaction. ${ }^{27,58}$ It is frequently assumed that their beneficial effect is mainly mediated by a positive change in the relatives' attitudes. ${ }^{59}$

It has been suggested that psychoeducation should be routinely provided to family members in order to keep them in contact with health services. It has been reported that psychoeducation reduces recurrence and improves adherence to medication received by the patient. It may also be the initial part of a more complex intervention, as would family intervention. Also, it allows the family to increase their knowledge and coping strategies. ${ }^{60}$

However, once the psychoeducation program is completed, studies show that effective treatment for patients with schizophrenia needs further monitoring and involvement of health professionals because both family and patients will have long-term problems, needing support and strategies to cope with these difficulties, and hence the service to the patient and family should be permanent. ${ }^{61}$ Negative attitudes toward the patient, such as high levels of expressed emotion (EE) involving criticism, over-involvement, and intrusiveness, ${ }^{62}$ and the attribution of symptoms to the patient's willpower have been shown to be related to worse prognosis of the illness. Vaughn and Leff ${ }^{63}$ showed that patients in families that show a high EE will have more relapses (48\%) than in families where the EE is low $(6 \%)$. Their results also confirmed the protective effect of medication and the presence of reduced social contact in families showing high EE. The isolated fact of belonging to a family with high EE does not guarantee a relapse in a safe patient with schizophrenia; 9 therefore, the consequences of the absence or presence of EE should be analyzed case by case.

Most current research regarding EE has extended to relationships between patients and psychiatric staff; it has been found that professionals who are more likely to be critical of their patients tend to believe that symptoms are within the patient's control, ${ }^{64}$ and this could influence the attitude of the family toward the patient.

The results of a study carried out by Carrà et $\mathrm{al}^{52}$ showed that neither the number of previous hospitalizations nor the duration of disease was associated with high EE. This occurred in patients whose psychosocial functioning seemed to have benefited by the previous access to formal education, and it could be considered as a protection factor. This last point reinforces again the need for cross-cultural comparisons about the subjective experience of distress and burden among caregivers, as a target for intervention. The latter is linked to reduced family stress and improved results for 
patient treatment. Family intervention may reduce levels of $\mathrm{EE}$ in the family, as well as improve the quality of life of both patients and relatives. ${ }^{2}$

Because of this, current family therapy programs focus not only on providing information about the illness and its management but also on promoting positive attitudes such as empathy and affective support and on changing verbal communications patterns between the family members. ${ }^{59}$ Recently, Kuipers et al proposed a cognitive model of caregiving in which they describe the main types of caregiving relationships in psychosis and the proposed family interventions. ${ }^{65}$ This model is based on caregiving appraisals and affective reactions in psychosis, which lead to a range of caregiver behaviors toward service users and toward mental health services, as well as to different caregiver outcomes. Therefore, these authors suggest that specific interventions should target these appraisals and their maintenance factors in order to improve the therapy outcomes.

In terms of the duration of the intervention, the literature indicates that the best results are obtained after three months of treatment; however, the effects may disappear after a few months if the therapy is not maintained. ${ }^{27-29,58}$ This is how the multidisciplinary care delivered to patients with schizophrenia not only helps to improve their psychiatric symptoms but also decreases the level of burden on the caregivers and promotes the integration of the family in the treatment. ${ }^{9}$

\section{Future challenges and solutions}

Despite the evidence of data supporting and confirming the importance and validity of family interventions, there are still obstacles to implementing them in the clinical setting. First, attending a family program conveys a series of demands to the caregivers, such as transportation (which also implies money), time, motivation, and energy. Also the stigma can cause relatives to quit. ${ }^{66}$ Another difficulty is the lack of preparation and specific training of therapists. The mental health structures providing this service within a system of mental health care are also altered. Also, several difficulties are associated with the need to understand the culture in which the family and the patient are living. Intercultural approaches could make important contributions to the adaptation of family interventions. ${ }^{52}$ Cost-and-effectiveness criteria also contribute to the scarce implementation because, most of the time, these interventions are estimated as highly expensive. However, they are expensive only if we consider the time needed to implement them. If we compare expenses and savings generated by the decrease of hospitalizations - approximately a third as indicated in most of the studies they should be considered as a low-cost intervention. ${ }^{67}$
Improvement and adaptation of the already existing therapies are also big challenges for both the clinical and scientific communities. As part of their role, some caregivers experience verbal and physical aggression and approximately one-third of caregivers report reactions indicative of posttraumatic stress disorder. ${ }^{68}$ One consequence of these reactions is adopting some maladaptive confronting styles, such as avoidance. Therefore, it is absolutely necessary to identify if these reactions are occurring in any of the members of the family and to adapt and incorporate specific techniques to overcome stress reactions and to improve coping strategies.

Another important area that needs further exploration in terms of the implementation of family interventions is early psychosis. During the early stages of the illness, patients are more likely to be living with their families of origin ${ }^{69}$ and families play a key role in ensuring medication adherence, and the care and well-being of the patient. Nonetheless, this particular period can be challenging for families as they struggle to make sense of the problems faced by their relative, including odd, unusual and embarrassing behaviors that may not always seem related to the illness. ${ }^{70}$ A recent meta-analysis conducted by Bird et $\mathrm{al}^{71}$ showed that family interventions in early psychosis significantly reduced relapse and readmission rates. It is very important that services have clear protocols for identifying those in need of family therapy in early psychosis.

Finally, the lack of research on family interventions for relatives of patients suffering from schizophrenia and severe comorbid medical conditions has to be highlighted. Medical comorbidity (eg, hypothyroidism, cardiovascular and pulmonary diseases, or diabetes) is common in schizophrenia and affects the quality of life and delivery of psychiatric and medical services. ${ }^{72}$ Primary care services and mental health services must consider all these comorbid medical conditions when treating schizophrenia patients and educate relatives on both treatment and prevention of these conditions, because they can exacerbate psychotic symptoms. ${ }^{73}$

\section{Conclusion}

A change in the opinion and attitudes of many mental health professionals in relation to family therapy is absolutely necessary. An integrated approach is only feasible if professionals work collaboratively and understand that schizophrenia is a mental health disorder that affects multiple areas of the patient and requires a comprehensive approach. This includes a patient's physical health - because it is well known that there is a relationship between schizophrenia and medical illnesses $^{74}$ - as well as mental health and social services. 
Mental health systems should include in their budget training resources for their professionals in order to provide a better service in the near future for both the patient and the caregivers. It is important that mental health services promote the participation of relatives in psychoeducational interventions, helping to build a proper family emotional environment that will benefit the caregivers-patient relationship and will improve the patient's functioning. Further work is required to identify key mediators of change when delivering family therapy in psychosis and to determine the most effective format and timing of the interventions..$^{58}$ Not all families require intensive support or structured interventions, but all families deserve to be offered the opportunity of being informed about the illness and to be provided with any resource that can improve their own and their relatives' well-being.

The responsibility of implanting family interventions is shared by several actors, where an important role is played not only by researchers and clinicians but also by government authorities of every country, which have the responsibility to meet the demands of health services and to create a fair and democratic society for all. Governments should be an active facilitator to enable these caregivers to have access to financial support to fulfill their role, promoting jobs outside the home by promoting reduced working days and supplying residences with specialized teams to take care of the patient while the relative takes a holiday to maintain a better quality of life. The challenge is established. Scientific knowledge reinforces the fact that better management is necessary to provide these caregivers with the opportunity to lead a healthy and full life.

\section{Acknowledgments}

This research was funded by Research Project 3730-14 of Universidad de Tarapacá and by Convenio de Desempeño UTA-MINEDUC.

\section{Disclosure}

The authors report no conflicts of interest in this work.

\section{References}

1. Weisman A. Integrating culturally based approaches with existing interventions for Hispanic/Latino families coping with schizophrenia. Psychotherapy. 2005;42(2):178-197.

2. Okpokoro U, Adams CE, Sampson S. Family intervention (brief) for schizophrenia. Cochrane Database Syst Rev. 2014;3:CD009802.

3. Barker S, Lavender T, Morant N. Client and family narratives on schizophrenia. J Ment Health. 2001;10:199-212.

4. Chadda R, Singh T, Ganguly K. Caregiver burden and coping. A prospective study of relationship between burden and coping in caregivers of patients with schizophrenia and bipolar affective disorder. Soc Psychiatry Psychiatr Epidemiol. 2007;42(11):923-939.
5. Clark R, Drake R. Expenditures of time and money by families of people with severe mental illness and substance use disorders. Community Ment Health J. 1994;30:145-163.

6. Addington J, Burnett P. Working with families in the early stages of psychosis. In: Gleesson J, McCorry P, editors. Psychological Interventions for Early Psychosis. Chichester: Wiley and Sons; 2004: 99-116.

7. McDonell MG, Short RA, Berry CM, Dyck DG. Burden in schizophrenia caregivers: impact of family psychoeducation and awareness of patient suicidality. Fam Process. 2003;42:91-103.

8. Hou S, Ke C, Su Y, Lung F, Huang C. Exploring the burden of the primary family caregivers of schizophrenia patients in Taiwan. Psychiatry Clin Neurosci. 2008;62(5):508-514.

9. Muela J, Godoy J. El estrés crónico en la esquizofrenia: la emoción expresada. Revista electrónica de Motivación y Emoción. 2001; 4(7):1-18.

10. Magliano L, Fiorillo A, De Rosa C, Malangone C, Maj M. Family burden in long-term disease: a comparative study in schizophrenia vs physical disorders. Soc Sci Med. 2005;61(2):313-322.

11. McCann TV, Luban DI, Clark E. First time primary caregivers' experience from young adults with first episode psychosis. Schizophr Bull. 2011;37(2):381-388.

12. Mueser KT, Webb C, Pfeiffer M. Family burden of schizophrenia and bipolar disorders: perceptions of relatives and professionals. Psychiatr Serv. 1996;47:507-511.

13. Wolthaus JE, Dingemans PM, Schene AH, et al. Caregiver burden in recent-onset schizophrenia and spectrum disorders: the influence of symptoms and personality traits. J Nerv Ment Dis. 2002;190: 241-247.

14. Ostman M, Kjellin L. Stigma by association: psychological factors in relatives of people with mental illness. Br J Psychiatry. 2002;181: 494-498.

15. Shibre T, Negash A, Kullgren G, et al. Perception of stigma among family members of individuals with schizophrenia and major affective disorders in rural Ethiopia. Soc Psychiatry Psychiatr Epidemiol. 2001; 36(6):299-303.

16. Struening EL, Perlick DA, Link BG, Hellman F, Herman D, Sirey JA. Stigma as a barrier to recovery: the extent to which caregivers believe most people devalue consumers and their families. Psychiatr Serv. 2001;52(12):1633-1638.

17. Castilla A, López M, Chavarría M, et al. La carga familiar en una muestra de pacientes esquizofrénicos en tratamiento ambulatorio. Rev Asoc Esp Neur. 1998;18:621-642.

18. Thara R, Kamath S, Kumar S. Women with schizophrenia and broken marriages-doubly disadvantaged? Part II: Family perspective. Int J Soc Psychiatry. 2003;49:233-240.

19. Stam H, Cuijpers P. Brief report: effects of family interventions on burden of relatives of psychiatric patients in the Netherland: a pilot study. Community Ment Health J. 2001;37:179-187.

20. Lauber C, Eichenberger A, Luginbühl P, Keller C, Rössler W. Determinants of burden in caregivers of patients with exacerbating schizophrenia. Eur Psychiatry. 2003;18:285-289.

21. Gutiérrez-Maldonado J, Caqueo-Urízar A, Kavanagh D. Burden of care and general health in families of patients with schizophrenia. Soc Psychiatry Psychiatr Epidemiol. 2005;40:899-904.

22. Caqueo-Urízar A, Gutiérrez-Maldonado J. Burden in families of patients with schizophrenia. Qual Life Res. 2006;15:719-724.

23. Colina A. Los cuidados no formales en el contexto de la salud mental comunitaria. En: Estigma, necesidades y cuidados no formales en personas con trastornos picóticos de larga evolución. Ponencia en el II Congreso Virtual de Psiquiatría, 2001.

24. Leal M, Sales R, Ibáñez E, Giner J, Leal C. Valoración de la sobrecarga en cuidadores informales de pacientes con esquizofrenia antes y después de un programa psicoeducativo. Actas Esp Psiquiatr. 2008;36(2):63-69.

25. Berglund N, Vahlne J, Edman A. Family intervention in schizophrenia. Impact on family burden and attitude. Soc Psychiatry Psychiatr Epidemiol. 2003;38:116-121. 
26. Riley G, Gregory N, Bellinger J, Davies N, Mabbott G, Sabourin R. Carer's education groups for relatives with a first episode of psychosis: an evaluation of an eight-week education group. Early Interv Psychiatry. 2011;5(1):57-63.

27. Chan SW, Yip B, Tso S, Cheng BS, Tam W. Evaluation of a psychoeducation program for Chinese clients with schizophrenia and their family caregivers. Patient Educ Couns. 2009;75(1):67-76.

28. Koolaee AK, Etemadi A. The outcome of family interventions for the mothers of schizophrenia patients in Iran. Int J Soc Psychiatry. 2010; 56(6):634-646.

29. Pitschel-Walz G, Leucht S, Bauml J. The effect of family interventions on relapse and rehospitalization in schizophrenia - a meta-analysis. Schizophr Bull. 2001;27:73-92.

30. Pilling S, Bebbington P, Kuipers E. Psychological treatment in schizophrenia. 1. Meta-analysis of family intervention and cognitive behaviour therapy. Psychol Med. 2002;32:763-782.

31. Pharaoh F, Rathbone J, Mari JJ. Family intervention for schizophrenia. Cochrane Database Syst Rev. 2003;(4):CD000088.

32. Magliano L, Fiorillo A, Fadden G, et al. Efectividad de una forma de intervención psicoeducativa sobre las familias de pacientes con esquizofrenia: resultados preliminares de un estudio sufragado por la Comisión Europea. World Psychiatry (ed. esp.). 2005;3:45-49.

33. Webb C, Pfeiffer M, Mueser KT, et al. Burden and well-being of caregivers for the severely mentally ill: the role of coping style and social support. Schizophr Res. 1998;34:169-180.

34. Ötsmann M, Hansson L, Anderson K. Family burden, participation in care and mental health - an 11-year comparison of the situation of relatives to compulsorily and voluntarily admitted patients. Int $J$ Soc Psychiatry. 2000;46:191-200

35. The WHOQOL Group. Study protocol for the World Health Organization project to develop a Quality of Life assessment instrument (WHOQOL). Qual Life Res. 1993;42(2):153-159.

36. Caqueo A, Urzúa A. Calidad de vida: una revisión teórica del concepto. Revista Terapia Psicológica. 2002;30(1):61-71.

37. Harvey K, Burns T. Relatives of patients with severe mental disorders: unique traits and experiences of primary, nonprimary, and lone caregivers. Am J Orthopsychiatry. 2003;73(3):324-333.

38. Argimon JM, Limon E, Vila J, Cabezas C. Health-related quality of life in carers of patients with dementia. Fam Pract. 2004;21(4):454-457.

39. Caqueo-Urizar A, Gutierrez-Maldonado J, Miranda-Castillo C. Quality of life in caregivers of patients with schizophrenia: a literature review. Health Qual Life Outcomes. 2009;7:84.

40. Foldemo A, Gullberg M, Ek AC, Bogren L. Quality of life and burden in parents of outpatients with schizophrenia. Soc Psychiatry Psychiatr Epidemiol. 2005;40(2):133-138.

41. Osman CB, Alipah B, Tutiiryani MD, Ainsah O. Depressive disorders and family functioning among the caregivers of patients with schizophrenia. East Asian Arch Psychiatry. 2010;20(3):101-108.

42. Mubarak A, Barber J. Emotional expressiveness and the quality of life of patients with schizophrenia. Soc Psychiatry Psychiatr Epidemiol. 2003;38:380-384

43. Espina A, González P. Intervenciones familiares en la esquizofrenia. Cambios en la sintomatología y el ajuste social. Rev Salud Ment. 2003; 26:51-58.

44. Grandón P, Jenaro C, Lemos S. Primary caregivers of schizophrenia outpatients: burden and predictor variables. Psychiatry Res. 2008; 158:335-343.

45. Kulhara P, Kate N, Grover S, Nehra R. Positive aspects of caregiving in schizophrenia: A review. World J Psychiatry. 2012;2(3): 43-48.

46. Kate N, Grover S, Kulhara P, Nehra R. Positive aspects of caregiving and its correlates in caregivers of schizophrenia: a study from North India. East Asian Arch Psychiatry. 2013;23:45-55.

47. Szmukler GI, Burgess P, Herrman H, Benson A, Colusa S, Bloch S. Caring for relatives with serious mental illness: the development of the Experience of Caregiving Inventory. Soc Psychiatry Psychiatr Epidemiol. 1996;31:137-148.
48. Nolan M, Grant G, Keady J. Understanding Family Care. A Multidimensional Model of Caring and Coping. Buckingham: Open University Press; 1996.

49. Lawton MP, Moss M, Kleban MH, Glicksman A, Rovine M. A twofactor model of caregiving appraisal and psychological well-being. J Gerontol. 1991;46:181-189.

50. Lawton MP, Rajagopal D, Brody E, Kleban MH. The dynamics of caregiving for a demented elder among black and white families. J Gerontol. 1992;47:S156-S164.

51. Kramer BJ. Gain in the caregiving experience: where are we? What next? Gerontologist. 1997;37:218-232.

52. Carrà G, Cazzullo $\mathrm{C}$, Clerici M. The association between expressed emotion, illness severity and subjective burden of care in relatives of patients with schizophrenia. Findings from an Italian population. BMC Psychiatry. 2012;12:140.

53. Mueser KT, Deavers F, Penn DL, Cassisi J. Psychosocial treatments for schizophrenia. Annu Rev Clin Psychol. 2013;9:465-497.

54. National Institute for Clinical Excellence. Schizophrenia (update). Clinical Guidelines CG82. NICE; 2009. Available from: http://www. guidance.nice.org.uk/CG82

55. Pharoah F, Mari J, Rathbone J, Wong W. Family intervention for schizophrenia. Cochrane Database Syst Rev. 2010;(12):CD000088.

56. Pilling S, Bebbington P, Garety P, Kuipers E, Geddes J, Martindale B. Meta-analysis of psychological treatment in psychosis I; family work and individual CBT. Psychol Med. 2002;32:763-782.

57. Giron M, Fernandez-Yanez A, Mana-Alvarenga S, Molina-Habas A, Nolasco A, Gomez-Beneyto M. Efficacy and effectiveness of individual family intervention on social and clinical functioning and family burden in severe schizophrenia: a 2-year randomized controlled study. Psychol Med. 2010;40(1):73-84.

58. Kulhara P, Chakrabarti S, Avasthi A, Sharma A, Sharma S. Psychoeducational intervention for caregivers of Indian patients with schizophrenia: a randomised-controlled trial. Acta Psychiatr Scand. 2009;119(6): 472-483.

59. Giron M, Nova-Fernandez F, Mana-Alvarenga S, et al. How does family intervention improve the outcome of people with schizophrenia? Soc Psychiatry Psychiatr Epidemiol. 2014:doi:10.1007/s00127-014-0942-9.

60. Sin J, Norman I. Psychoeducational interventions for family members of people with schizophrenia: a mixed-method systematic review. J Clin Psychiatry. 2013;74(12):e1145-e1162.

61. Carrà G, Montomoli C, Clerici M, Cazzullo C. Family interventions for schizophrenia in Italy: randomized controlled trial. Eur Arch Psychiatry Clin Neurosci. 2007;257:23-30.

62. Butzlaff RL, Hooley JM. Expressed emotion and psychiatric relapse: a meta-analysis. Arch Gen Psychiatry. 1998;55:547-552.

63. Vaughn C, Leff J. The measurement of expressed emotion in the families of psychiatric patients. Br J Soc Clin Psychol. 1976;15:157-165.

64. Winship G. Increasing awareness of expressed emotion in schizophrenia: an evaluation of a staff training session. $J$ Psychiatr Ment Health Nurs. 2011;18:277-280.

65. Kuipers E, Onwumere J, Bebbington P. A cognitive model of caregiving in psychosis. Br J Psychiatry. 2010;196:259-265.

66. McFarlane W, Dixon L, Luckens E, et al. Family psychoeducation and schizophrenia: a review of the literature. J Marriage Fam. 2003;29: 223-245.

67. Jonson D. The major barriers to implement the finding. In: Oral Communication. 6th World Congress for Psychosocial Rehabilitation, May 2-5, 1998; Hamburg, Germany.

68. Loughland CM, Lawrence G, Allen J, et al. Aggression and trauma experiences among carer relatives of people with psychosis. Soc Psychiatry Psychiatr Epidemiol. 2009;44:1031-1040.

69. Fisher H, Theodore K, Power P, et al. Routine evaluation in first episode psychosis services: feasibility and results from the MiData project. Soc Psychiatry Psychiatr Epidemiol. 2008;43:960-967.

70. Onwumere J, Bebbington P, Kuipers E. Family interventions in early psychosis: specificity and effectiveness. Epidemiol Psychiatr Sci. 2011; 20(2):113-119. 
71. Bird V, Premkumar P, Kendall T, Whittington C, Mitchell J, Kuipers E. Early intervention services, cognitive-behavioural therapy and family intervention in early psychosis: systematic review. Br J Psychiatry. 2010;197:350-356.

72. Carney CP, Jones L, Woolson RF. Medical comorbidity in women and men with schizophrenia: a population-based controlled study. J Gen Intern Med. 2006;21(11):1133-1137.
73. Hall RC, Gardner ER, Popkin MK, Lecann AF, Stickney SK. Unrecognized physical illness prompting psychiatric admission: a prospective study. Am J Psychiatry. 1981;138:629-635.

74. Torres-González F, Ibanez-Casas I, Saldivia S, et al. Unmet needs in the management of schizophrenia. Neuropsychiatr Dis Treat. 2014;10: 97-110.

\section{Publish your work in this journal}

Neuropsychiatric Disease and Treatment is an international, peerreviewed journal of clinical therapeutics and pharmacology focusing on concise rapid reporting of clinical or pre-clinical studies on a range of neuropsychiatric and neurological disorders. This journal is indexed on PubMed Central, the 'PsycINFO' database and CAS, and is the official journal of The International Neuropsychiatric Association (INA). The manuscript management system is completely online and includes a very quick and fair peer-review system, which is all easy to use. Visit http://www.dovepress.com/testimonials.php to read real quotes from published authors.

Submit your manuscript here: http://www.dovepress.com/neuropsychiatric-disease-and-treatment-journal 